\title{
The effect of medical clowns on blood pressure measurement in children presenting to the emergency department
}

\author{
Omer Raviv* \\ Meir Medical Center, Kfar Saba, Israel
}

\begin{abstract}
Blood pressure is measured in the triage among most children presenting to the Pediatric ED. There are many barriers to obtaining an accurate BP measurement in children, including inappropriate technique, lack of equipment, but also - pain, and the child's uncooperativeness or anxiety. The later may even be accentuated in the ED setting.

These factors may lead to falsely elevated BP reading, which may provoke significant anxiety in children and families and possibly lead to a cascade of unnecessary investigation and consultations.

Additionally, an abnormal measured BP value routinely mandate an additional measurement attempt which itself may cause anxiety and restlessness among children, especially toddlers and infants and prolongs the entire triage process.
\end{abstract}

The Second Task Force Report on Blood Pressure Control in Children does emphasize blood pressure measurement in a quiet environment, using a proper size cuff, and using appropriate technique [1]. Ideally, BP should be measured in a quiet setting in a relaxed child, with several measurements taken at each. This scenario is nearly impossible in the emergency department, as demonstrated in a previous retrospective study that indicated that although $\mathrm{BP}$ was elevated in $52 \%$ of children who underwent triage blood pressure measurement in the $\mathrm{ED}$, the measurement was repeated in the in only $38 \%$ [2].

In the past decade, there has been a rapid growth in the presence of therapeutic clowns in hospitals, particularly in pediatric settings [3]. a recent study conducted in a PED in Israel has demonstrated the benefit of medical clowns in diminishing pain and anxiety during painful procedures being performed on children in the ED [4].

Previous studies conducted in our institute have demonstrated a beneficial effect on vital signs [blood pressure and heart rate] as a sign of decreased anxiety following the incorporation of a medical clown in a multisystem team caring for pediatric patient $[5,6]$.

Blood pressure measurement among pediatric patients, although is not a painful procedure, may cause a stressful reaction, prolonging the entire process and requiring additional attempts - making it a time consuming task in a busy ED.

A beneficial effect of a medical clown on the pediatric patient by decreasing the stress reaction and may shorten the entire process, and, in terns, making it much more efficient.

We are unaware of any studies conducted related to the effect of the use of medical clowns during ED blood pressure measurements in the triage.
AIMS: we speculate that the incorporation of a medical clone during the procedure of blood pressure measurement in the triage will shorten the procedure and decrease the number of attempts, making it much more efficient.

\section{Methodology}

200 children presenting to the pediatric ED will be randomly assigned to two groups The study group - children will undergo the routine triage process, including BP measurement with the presence of the medical clown.

The control group of children will undergo the same process without the intervention of a medical clown.

The medical clown team in Meir pediatric department is constant and each uses the same methodology while working in the ED.

The time of blood pressure measurement will be taken for every attempt and the number of attempts of blood pressure measurement will be recorded.

The time of measurements, number of trials and the medical measurements will be documented along with demographic background.

Additionally, a visual analog scale [VAS] for pain will be completed during the process in both groups.

${ }^{\star}$ Correspondence to: Omer Raviv, Meir Medical Center, Kfar Saba, Israel, Tel: 972528822052, E-mail: omerol@walla.co.il

Received: May 15, 2018; Accepted: June 15, 2018; Published: June 19, 2018 
VAS score is a pain assessment scale from $0-10$, while 0 indicate no pain, relaxed child and 10 indicate suffering and severe pain.

A written parental agreement will be obtained - following a given oral and written explanation.

The time for data collection is estimated for 6 months

\section{Inclusion criteria}

Children aged $0-18$ years presenting to the ED.

\section{Exclusion criteria}

1. Serious medical condition requiring emergent medical care or resuscitation

2. Parental refusal to participate in the study

\section{Project stuffing}

During the study period - the prescience of a medical clown in the Pediatric ED Ttriage will be required.

Additional personal will be required for parent's recruitment, study assignment and time and attempts recording. No professional or medical experience is required.

\section{Budget}

We estimate that to fulfill the aim of gatherings data within 2-3 months we require at least 6-8 clown hours per week.

\section{References}

1. Task Force on Blood Pressure Control in Children: Report of the Second Task Force on Blood Pressure Control in Children. National Heart, Lung and Blood Institute, Bethesda, MD. Pediatrics 79: 1-25. [Crossref]

2. Silverman MA, Walker AR, Nicolaou DD, Bono MJ (2000) The frequency of blood pressure measurements in children in four EDs. Am J Emerg Med 18: 784-788. [Crossref]

3. Bertini M, Isola E, Paolone G, Curicio G (2011) Clowns benefit children hospitalized for respiratory pathologies. Evid Based Complement. Alternat Med: 879125.

4. Wolyniez I, Rimon A, Scolnik D, Gruber A (2013) The Effect of Medical Clown on Pain During Intravenous Access in the Pediatric Emergency Department: A Randomized Prospective Pilot Study. Clinical Pediatrics. 52: 1168-1172. [Crossref]

5. Uziel Y, Chapnik G, Rotchild M, Tauber T, Press J, et al. (2008) Nitrous oxide sedation for intra-articular injrctions in juvenile idiopathic arthritis. Pediatric Rheumatology 6: 1. [Crossref]

6. Oren-Ziv A, Hanuka P, Rotchild M, Gluzman A, Uziel Y (2012) Medical clowns dream doctors are important team members in the treatment of young children with juvenile idiopathic arthritis. Harefuah 151: 332-334, 380, 379. [Crossref]

Copyright: (C2018 Raviv O. This is an open-access article distributed under the terms of the Creative Commons Attribution License, which permits unrestricted use, distribution, and reproduction in any medium, provided the original author and source are credited. 